exposure to ELF-MF showed a positive association with follicular lymphoma.

Conclusions In this large prospective cohort study, we found indications of an association of ELF-MF exposure with AML and follicular lymphoma, but not with other types of cancer. These results warrant further research in the possible association of ELF-MF and haematopoietic cancers.

\section{EFFECTS OF MRI RELATED MAGNETIC FIELDS ON COGNITIVE PERFORMANCE}

'L E van Nierop, 'Slottje, 'van Zandvoort, '2 de Vocht, 'Kromhout. 'Utrecht University, Utrecht, Nederland; 'niversity of Manchester, Manchester, United Kingdom

\subsection{6/oemed-2013-101717.244}

Objectives The advances in modern medicine introduced stronger MRI systems up to 9.4 Tesla and a broader area of application. Since the use of stronger imaging systems the amount of reported side effects has increased. Among these symptoms are nausea, dizziness, fatigue sleeplessness, concentration problems and a metallic taste. An earlier study showed also negative effects of movement in the magnetic fields on concentration, visuo-spatial orientation and postural body sway.

Exposure to higher field strengths could have consequences for professions in medical settings where workers are repeatedly exposed. Especially for surgeons and radiologists working close to MRI systems need to maintain a high level of accuracy in performance and cognitive function. Better insight in acute effects on neurocognitive functions, will form a basis for the design of relevant protective and precautionary control measures.

In the present study we try to gain more insight which kind of magnetic fields are responsible for evoking the cognitive effects. We separate between the heterogeneous static magnetic field (SMF) around the scanner and movement induced timevarying magnetic fields (TVMF) within the static magnetic field. Methods We used a double blind randomised cross over design in which thirty six healthy volunteers underwent four test sessions. The two exposure conditions were near a 7 T MRI system with personal SMF exposure of 1.0 Tesla. In one of these conditions subjects had to make standardised head movements before every task which induced additional TVMF of $2.4 \mathrm{~T} / \mathrm{s}$. Of the two corresponding sham conditions $(0 \mathrm{~T})$ one was with and one without additional head movements. Cognitive tasks which showed an effect in previous studies were assessed.

Results Results of this study suggests different effects from SMF and TVMF on cognition.

\section{Session: 4. Occupational cancer}

\section{RISK OF EIGHT TYPES OF CANCER AND CLEANING- RELATED EXPOSURES IN A CASE-CONTROL STUDY}

'D V Vizcaya, 'J L Lavoué, ${ }^{2} \mathrm{D}$ B Bégin, 'J $\mathrm{P}$ Javier, ' $\mathrm{L}$ R Richardson, ${ }^{1} \mathrm{M}$ Rivera, IJ S Siemiatycki. ' CRCHUM, Montreal, Canada; ${ }^{2}$ Département de santé environnementale et santé au travail. Université de Montréal, Montreal, Canada

\subsection{6/oemed-2013-101717.245}

Objectives To evaluate the possible associations between occupational exposure to cleaning products and cancers of the following sites: bladder, pancreas, prostate, colon, stomach, kidney, non-Hodgkin's lymphoma, and lymphoid tissue (Hodgkin's and Non-Hodgkin's lymphomas and myelomas combined).
Methods We conducted a case-control study of occupational exposures and cancer in Montreal including 3730 cancer cases and 533 population controls. Occupational exposure to a set of agents was evaluated using a combination of subject-reported job history and expert assessment. We evaluated the association between each of eight cancer sites and each of eight cleaningrelated occupations, and each of seven cleaning-related exposures (ammonia, hypochlorite, spray gases, waxes/polishes, caustic solutions, chlorine, and cleaning agents as a class). Analyses were done using multivariate logistic regression.

Results In general, we found no increased risk of cancer for the evaluated occupations as compared to never cleaners with the exceptions of pancreatic cancer (Odds Ratio; 95\% CI (OR): 3.0; 1.3-6.8) and cancers of lymphoid origin (lymphomas and myelomas) (OR: $2.1 ; 1.1-4.1$ ) in relation to long term employment as 'janitors and cleaners'. Among specific agents, we found an indication of excess risk for substantial exposure to ammonia and hypochlorite with a pooled set of lymphoid cancers (Non-Hodgkin's lymphoma, Hodgkin's lymphoma and myeloma) (OR: 2.2; $1.2-4.0$ and $2.0 ; 1.0-4.0$, respectively). For the rest of the agents and cancer sites evaluated we found no evidence of increased risk.

Conclusions Overall, our results do not support a clear association between occupational exposure to cleaning products or cleaning-related occupations and the examined cancer sites, with the exception of a potential link between lymphoid cancers (Hodgkin's and Non-Hodgkin's lymphomas and myeloma) and exposure to ammonia and to hypochlorite. Elevated ORs among janitors and cleaners should be further explored. Our results must be interpreted in a context of multiple hypotheses testing.

\section{CHEMICAL EXPOSURE AND CANCER MORTALITY IN A FRENCH COHORT OF URANIUM PROCESSING WORKERS}

${ }^{1} S$ Zhivin, ${ }^{1}$ Laurier, ${ }^{1}$ Caër-Lorho, ${ }^{2}$ Acker, ${ }^{1}$ Guseva Canu. ${ }^{1}$ Institut de Radioprotection et de Sûreté Nucléaire (IRSN), Fontenay-aux-Roses, France; ${ }^{2}$ AREVA NC Medical Coordination Section, Paris, France

\subsection{6/oemed-2013-101717.246}

Objectives Besides radiation, nuclear workers can be exposed to a variety of chemical hazards. We examined the effect of chemical exposure on cancer mortality of French nuclear workers at the AREVA NC Pierrelatte establishment.

Methods A cohort of 2,897 uranium processing workers employed for at least 6 months was followed up from 1968 to 2006. Exposures to uranium and potentially carcinogenic chemicals were assessed using plant-specific job-exposure matrix. Lung, lympho haematopoietic, kidney and bladder, brain and other central nervous system (BCNS), and prostate cancers mortality hazard ratios (HRs) were estimated for each chemical exposure using Cox regression models stratified on sex and calendar period, and adjusted for socio-economic status. An effect of co-exposures to uranium and other chemicals was examined using additional adjustments.

Results Exposure to aromatic solvents was associated with increased risk of BCNS malignancies after adjustment for other chemicals $(\mathrm{HR}=6.53,95 \% \mathrm{CI}=1.14-37.41)$ and other chemicals and uranium $(\mathrm{HR}=7.26,95 \% \mathrm{CI}=0.90-58.19)$. Significantly elevated risk of lung cancer related to exposure to chromium (VI) compounds was also observed, based on only two deaths among exposed workers.

Conclusions Exposure to aromatic solvents was associated with the risk of BCNS cancers. The latter risk might be increased by 